\title{
Predictive value of microRNA-10b expression in peripheral blood mononuclear cells in evaluating short- and long-term efficacy of chemotherapy for patients with advanced non-small-cell lung cancer
}

\author{
Y.-L. YANG ${ }^{1}$, W. WANG ${ }^{2}$, L.-P. XU ${ }^{1, *}$ \\ ${ }^{1}$ Department of Cardiothoracic Surgery, the Affiliated Hospital of Beihua University, Jilin 132001, P.R. China; ${ }^{2}$ Clinical Research Institute Office, \\ the Central Hospital of Jilin City, Jilin 132011, P.R. China \\ *Correspondence: xuluping_XLP2017@163.com
}

Received January 10, 2017 / Accepted November 14, 2017

\begin{abstract}
The expression profile in peripheral blood mononuclear cells (PBMCs) from advanced non-small-cell lung cancer (NSCLC) patients and the role of microRNAs (miRs) in NSCLC remain unclear. Herein, the present study aims to investigate predictive value of miR-10b expression in PBMCs in evaluating short- and long-term efficacy of chemotherapy for NSCLC patients. A total of 194 advanced NSCLC patients were selected as the NSCLC group and 199 healthy individuals were recruited as a control group. Reverse transcription quantitative polymerase chain reaction (RT-qPCR) was performed to determine the miR-10b expression in PBMCs. The patients with advanced NSCLC were treated with chemotherapy, and the relationship between miR-10b expression in PBMCs and the clinicopathological characteristics, clinical response to chemotherapy and prognosis were analyzed. The values of miR-10b in diagnosis and prediction of clinical response to chemotherapy before chemotherapy were detected by receiver operating characteristic (ROC) curve analysis, the clinical response to chemotherapy by logistic regression analysis, and the risk factors of prognosis in NSCLC patients by Cox regression analysis. Compared with the control group, miR-10b in PBMCs was highly expressed in the NSCLC group. The area under the curve (AUC) of miR-10b expression in the diagnosis of NSCLC was 0.967 , with sensitivity and specificity of $85.10 \%$ and $99.5 \%$, respectively. MiR-10b expression was related to lymph node metastasis (LNM), distant metastasis, pathological types and differentiation. The AUC of miR-10b expression in the prognosis of advanced NSCLC was 0.793 , with sensitivity and specificity of $75.20 \%$ and $76.62 \%$, respectively. Logistic regression analysis showed that clinical response to chemotherapy was significantly influenced by miR-10b expression, distant metastasis and LNM. Cox regression analysis showed that the high miR-10b expression, smoking, LNM and distant metastasis were risk factors of prognosis for advanced NSCLC patients. The present study offers intriguing new perspectives based on evidence that miR-10b expression in PBMCs has predictive value for the tumor response to chemotherapy and prognosis for advanced NSCLC patients.
\end{abstract}

Key words: microRNA-10b, advanced non-small-cell lung cancer, peripheral blood mononuclear cells, chemotherapy, differentiation, prognosis

Lung cancer, a prominent malignant tumor, has become the first leading cause of death throughout the world, and can be classified into two subtypes, small cell lung cancer (SCLC) and non-small-cell lung cancer (NSCLC) [1, 2]. NSCLC becomes the most commonly seen type of lung cancer as it accounts for $80 \%$ in all lung cancer cases [3]. Treatment methods for patients with stage III NSCLC consist of surgery, radiotherapy and chemotherapy, with cure rates reaching $10 \%$ to $25 \%$. For patients with stage IV NSCLC, it is primarily palliative chemotherapy combined with palliative radiotherapy or surgery controlling symptomatic distant tumor localization [4]. Additionally, smoking, operative methods, lymph node dissection, and blood loss are significantly related to postoperative complications [5]. The 5-year survival rate of patients with advanced NSCLC stage is very poor, and advanced stage NSCLC patients show poor results in long-term survival [6]. Even with the disappointing clinical results, there is an urging demand for effective diagnostic strategies to early identify high-risk populations faster and earlier, thus decreasing the mortality rate in NSCLC patients, as well as predictive factors for prognosis to manage the outcomes of the treatment [7]. 
Nowadays, upcoming evidence indicates that microRNAs (miRs), especially conserved non-coding RNAs, are diffusely used in the NSCLC occurrence and development [8].

MiRs are small, single-stranded non-coding RNA molecules with a length of 19-23 nucleotides, which can modulate gene expression [9]. MiRs, acting as tumor suppressors or oncogenes, play decisive roles in the occurrence and development of cancer with abnormal expressions [10]. It has been proven that MiR-10b plays critical roles in cancer progression in a variety of cancers, such as colorectal cancer, breast cancer and pancreatic cancers [11-13]. As a tumor enhancer, miR-10b is expressed in the NSCLC cell line A549, reported as a potential target for interventional therapy for NSCLC [14]. Furthermore, miR-10b is over-expressed in metastatic cancer cells, suggesting that miR-10b overexpression may enhance the invasion and metastatic potential of cancer [11]. In addition, miRs in peripheral blood mononuclear cells (PBMCs) in patients are found to be associated with malignant tumors and might be therefore used as cancer biomarkers $[15,16]$. However, limited researches pay attention to the value of miR-10b expression in PBMCs for the prognosis of NSCLC. Thus, our study aims to investigate the predictive value of miR-10b expression in PBMCs in tumor response to chemotherapy and prognosis for patients with advanced NSCLC, which mainly refers to the short- and long-term efficacy with a 5-year follow-up.

\section{Patients and methods}

Ethical statement. This study was approved by the Ethics Committee of the Affiliated Hospital of Beihua University. All patients signed informed consent in written form for this research.

Study subjects. From August 1998 to July 2006, 194 patients diagnosed with advanced NSCLC in the Affiliated Hospital of Beihua University were selected as the NSCLC group. Inclusion criteria: 1) patients with advanced NSCLC through pathological diagnosis; 2) patients without any other organ dysfunction, or disease requiring hospitalization; 3) patients in stage IV and those who had not received surgery or chemotherapy in stage III, based on the World Health Organization (WHO) histologic classification system of tumor and the American Joint Committee on Cancer (AJCC) staging manual [17]; 4) patients without contraindications to chemotherapy; 5) patients with complete medical records, and all patients without receiving chemotherapy and biotherapy before sampling. Exclusive criteria: 1) patients with other malignant tumors or with a history of malignant tumors; 2) patients who had received surgery and chemotherapy; 3) patients with congenital diseases, hereditary diseases, autoimmune diseases and cardiovascular diseases; 4) patients who were unable to get in touch. According to the clinical and pathological data in the NSCLC group, there were 120 patients aged $<60$ years and 74 patients aged $\geq 60$ years with mean age of $59.9 \pm 10.1$ years; the patients consisted of 113 males and 81 females, 117 patients with smoking history and 77 patients without smoking history; there were 80 patients with low and moderate differentiation, 114 patients with highly differentiated tumors according to the degree of tumor differentiation; there were 102 patients with distant metastases, 92 patients without distant metastasis, 86 patients with lymph node metastasis (LNM), and 108 patients without LNM; 119 patients with squamous cell carcinoma, 61 patients with adenocarcinoma and 14 patients with other types of cancer on the basis of different pathological types. Beside, NSCLC tissues and paracancerous tissues located more than $3 \mathrm{~cm}$ from the tumor tissue of 48 advanced NSCLC patients (30 males and 18 females) were collected for waxing and storage. There were 199 healthy individuals randomly selected in the same area as the control group, 87 patients aged $<60$ years and 112 patients aged $\geq 60$ years with mean age of $59.2 \pm 9.2$ years. There were 139 males and 60 females, 106 patients with smoking history and 93 patients without smoking history. No significant difference in age, gender and smoking history between the NSCLC and control groups (all $\mathrm{p}>0.05$ ).

Sample collection. Before and after four cycles of chemotherapy, peripheral blood samples ( $5 \mathrm{ml}$ each case) were collected by ethylenediamine tetra-acetic acid (EDTA) anticoagulant tube in the NSCLC and control groups. PBMCs were isolated from peripheral blood with lymphocyte separating liquid (07801, StemCells, Inc. California, USA), fully lysed with $1 \mathrm{ml}$ Trizol (15596-026, Invitrogen, Carlsbad, CA, USA) and kept at $-80^{\circ} \mathrm{C}$. The RNA was extracted from PBMCs when being used for avoiding RNA degradation caused by repeated freezing and thawing.

Reverse transcription quantitative polymerase chain reaction (RT-qPCR). Total RNA was extracted from the PBMCs using the miRNeasy Mini Kit (217004, Qiagen, Hilden, Germany). The concentration and purity of the RNA were detected by using ultraviolet spectrophotometry through optical density (OD) values at wavelengths of $260 \mathrm{~nm}$ and $280 \mathrm{~nm}$. The OD260/OD280 ratio between 1.7 and 2.1 indicates a high purity of the RNA. The cDNA template was synthesized by reverse transcription using a PCR amplification instrument (ABI Company, Oyster Bay, NY, USA). The RT-qPCR was conducted using ABI 7500 Real-Time PCR instrument. The reaction conditions were as follows: pre-denaturation at $95^{\circ} \mathrm{C}$ for $10 \mathrm{~min}, 40$ cycles of denaturation at $95^{\circ} \mathrm{C}$ for $10 \mathrm{~s}$, annealing at $60^{\circ} \mathrm{C}$ for $20 \mathrm{~s}$, extension at $72^{\circ} \mathrm{C}$ for $34 \mathrm{~s}$. The reaction system consisted of $10 \mu \mathrm{l}$ of the SYBR Premix Ex Taq ${ }^{\text {TM II }}, 0.8 \mu$ l of Forward Primer $(10 \mu \mathrm{M}), 0.8 \mu \mathrm{l}$ of Reverse Primer $(10 \mu \mathrm{M}), 0.4 \mu \mathrm{l}$ of the ROX Reference Dye, $2.0 \mu \mathrm{l}$ of cDNA template and $6.0 \mu \mathrm{l}$ of sterile distilled water. The forward primer sequence of miR-10b was 5'-GGATACCCTGTAGAACCGAA-3', the reverse primer sequence of miR-10b was 5'-CAGTGCGTGTCGTGGAGT-3'. The forward primer sequence of reference gene U6 was 5'-TGGGGTTATACATTGTGAGAGGA-3', the reverse primer sequence of U6 was 5'-GTGTGCTACGGAGTTCA- 
GAGGTT-3'. PCR results were analyzed by the Opticon Monitor software version 3.0 (Bio-Rad Laboratories, Inc., Hercules, CA, USA). The threshold was manually set in the lowest point of parallel rise in the logarithmic amplification curve, and the threshold cycle $(\mathrm{Ct})$ value was analyzed using $2^{-\Delta \Delta \mathrm{Ct}}$ method. The proportions of the target gene expression between the NSCLC group and the control group were expressed by $2^{-\Delta \Delta \mathrm{Ct}}$, the formula is as follows: $\Delta \Delta \mathrm{Ct}=\Delta \mathrm{Ct}_{\text {experimental group }}-\Delta \mathrm{Ct}_{\text {control group, }}$ which is $\Delta \mathrm{Ct}=\mathrm{Ct}_{\mathrm{miR}}-$ $\mathrm{Ct}_{\mathrm{U} 6}$. The experiment was repeated three times.

Chemotherapy regimens and efficacy evaluation. The first-line chemotherapy regimen was used for the patients with advanced NSCLC. Patients received a paclitaxel + cisplatin regimen with intravenous infusion of paclitaxel $\left(135-175 \mathrm{mg} / \mathrm{m}^{2}\right)$ and cisplatin $\left(70-80 \mathrm{mg} / \mathrm{m}^{2}\right)$ on day 2 , 3 and 4, respectively; and navelbine (NVB) $\left(25 \mathrm{mg} / \mathrm{m}^{2}\right)$ on day 1 and 8 ; which was repeated every three weeks in one chemotherapy cycle [18].

After four cycles of chemotherapy [18], according to the Response Evaluation Criteria In Solid Tumors (RECIST 1.1) $[19,20]$, the results were categorized as complete response $(\mathrm{CR})$, partial response $(\mathrm{PR})$, progressive disease $(\mathrm{PD})$ and stable disease (SD). CR and PR were defined as overall response (OR), $\mathrm{PD}$ and $\mathrm{SD}$ were treated as non-remission or ineffective. Computed tomography (CT) scan was performed after four cycles of chemotherapy to evaluate the clinical response to chemotherapy.

Follow-up. The follow-up was conducted by telephone, outpatient and medical records and ended in September 2016 with 5 cases lost during the time. The follow-up rate was $97.42 \%$. Progression free survival (PFS) is defined as the time between treatment initiation and tumor recurrence or death from any cause.

Statistical analysis. The statistical analysis was conducted using SPSS 21.0 (IBM Corp. Armonk, NY, USA). The measurement data were presented by the mean \pm standard deviation (SD). Differences between two groups were compared by t-test. Receiver operating characteristic (ROC) curve was used to evaluate the prognostic value of
miR-10b expression in PBMCs with chemotherapy for advanced NSCLC patients. The logistic regression analysis was conducted to analyze the influencing factors of the clinical response to chemotherapy for advanced NSCLC patients. Probability of patients' survival time was presented by using Kaplan-Meier curve and Log-rank test was applied to detect the survival curve difference between groups. The Cox regression model was applied to analyze the prognostic factors of advanced NSCLC patients.A p-value $<0.05$ was considered statistically significant.

\section{Results}

MiR-10b is highly expressed in NSCLC tissues and PBMCs of advanced NSCLC patients. The relative expression of miR-10b in NSCLC tissues was obviously higher while miR-100 was lower than that in paracancerous tissues (both $\mathrm{p}<0.05)$. The relative expression of miR-10b in PBMCs of the NSCLC group was higher while the miR-100 was lower than that in the control group (both $\mathrm{p}<0.05$ ) (Figure 1).

Correlation between miR-10b expression in PBMCs and clinicopathological features of advanced NSCLC patients. Association between miR-10b expression in PBMCs and the clinicopathologic features of the patients with advanced NSCLC is depicted in Table 1. Patients with LNM showed significantly higher miR-10b expression than those without $(3.39 \pm 0.97$ vs. $2.59 \pm 0.94)(\mathrm{p}<0.05), \mathrm{miR}-10 \mathrm{~b}$ expression in patients with distant metastasis was evidently upregulated than in patients without distant metastasis $(3.62 \pm 0.85$ vs. $2.51 \pm 0.91)(\mathrm{p}<0.05), \mathrm{miR}-10 \mathrm{~b}$ expression in patients with low-moderate tumor differentiation was prominently enhanced than in patients with high differentiation $(2.85 \pm 1.04$ vs. $3.30 \pm 0.98)$, miR-10b expression in patients with squamous cell carcinoma was remarkably escalated than in patients with adenocarcinoma and other pathological types $(3.29 \pm 0.98$ vs. $2.66 \pm 1.02,3.29 \pm 0.98$ vs. $2.51 \pm 0.94)$, and miR-10b expression in patients with smoking history was markedly increased than in patients without smoking history (3.17 \pm 0.99 vs. $2.84 \pm 1.08)$. There was no significant

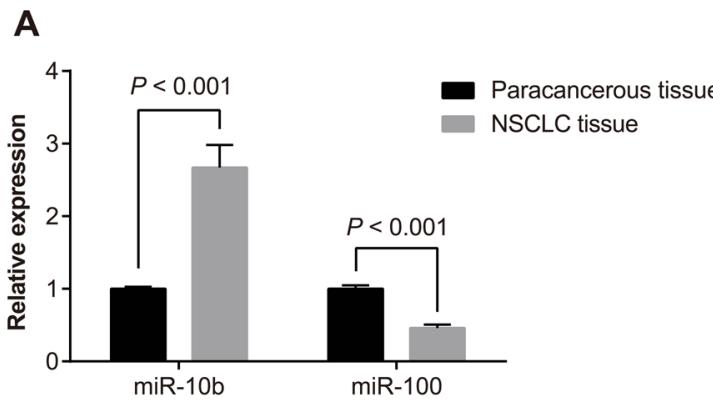

B

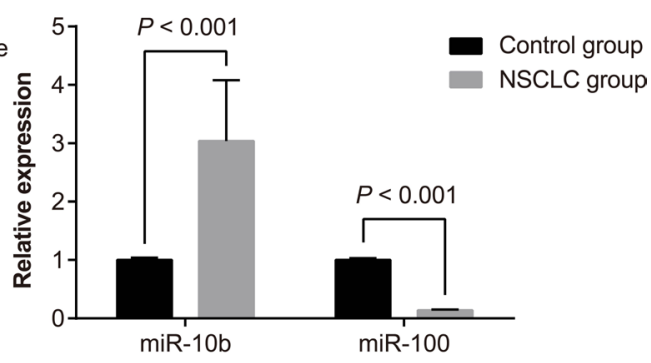

Figure 1. Expression of miR-10b and miR-100 in PBMCs in the NSCLC and control groups, NSCLC tissues and paracancerous tissues. A) expression of miR-10b and miR-100 in the NSCLC tissues and paracancerous tissues; B) expression of miR-10b and miR-100 in PBMCs; NSCLC; non-small-cell lung cancer; PBMCs, peripheral blood mononuclear cells; miR-10b, microRNA-10b; miR-100, microRNA-100. 
association between miR-10b expression and age, gender (all $\mathrm{p}>0.05)$.

Correlation between miR-10b expression in PBMCs and the tumor response to chemotherapy. The relative expression of miR-10b in PBMCs before and after four cycles of chemotherapy was $3.04 \pm 1.04$ and $2.71 \pm 0.99$, respectively. After four cycles of chemotherapy, miR-10b expression was markedly diminished when compared with the time before chemotherapy $(\mathrm{p}<0.05)$. Among 194 patients, there were 23 patients with CR, 54 patients with $\mathrm{PR}, 48$ patients with $\mathrm{PD}$, 69 patients with SD, which indicated that chemotherapy was effective for 77 patients (39.69\%). The miR-10b expression in the effective group $(\mathrm{CR}+\mathrm{PR})$ before chemotherapy and after four cycles of chemotherapy was $2.28 \pm 0.86$ and $1.94 \pm 0.79$, respectively, and in the ineffective group (SD+PD) it was $3.54 \pm 0.82$ and $3.22 \pm 0.75$, respectively. The results indicated that miR-10b expression in the effective group was dramatically elevated after four cycles of chemotherapy than before chemotherapy $(\mathrm{p}<0.05)$. The relative expression of miR-10b was significantly lower in the effective group before chemotherapy and after four cycles of chemotherapy than in the ineffective group (all p<0.05) (Figure 2).

Predicative values of miR-10b for the diagnosis and efficacy of chemotherapy for patients with advanced NSCLC. ROC curve of miR-10b expression in the PBMCs of the NSCLC and control groups before chemotherapy is shown in Figure 3A. The area under the curve (AUC) was calculated to assess the diagnostic efficacy of miR-10b in patients with NSCLC. The ROC curve demonstrated that AUC value of miR-10b in the diagnosis of NSCLC was 0.967 (95\% CI: 0.952-0.982), suggesting a good predicative value. The cutoff value was 1.98, the diagnostic sensitivity and specificity of miR-10b in the diagnosis of NSCLC were $85.10 \%$ and $99.50 \%$.

Figure $3 \mathrm{~B}$ shows the ROC curve of miR-10b expression in the PBMCs of the effective and ineffective groups before chemotherapy. The AUC value of miR-10b was 0.793 (95\% CI: 0.725-0.861), indicating a good predictive value of miR-10b for the efficacy of chemotherapy. The cutoff value was 2.87 , the diagnostic sensitivity was $75.20 \%$ and specificity was $76.62 \%$.
Influencing factors for the chemotherapy efficacy for advanced NSCLC patients by logistic regression analysis. Logistic regression analysis was performed with the efficacy of chemotherapy for advanced NSCLC patients as the dependent variable, and the clinicopathological features and the expression of miR-10b as the independent variable (Table 2). The results proved that distant metastasis, LNM, smoking history and miR-10b expression had a significant influence on the efficacy of chemotherapy $(\mathrm{p}<0.05)$. There was no significant difference in age, gender, tumor differentiation and pathological types (all p>0.05).

Table 1. Association between the relative expression of miR-10b and the clinicopathological features for advanced NSCLC patients.

\begin{tabular}{|c|c|c|c|}
\hline $\begin{array}{l}\text { Clinicopathological } \\
\text { data }\end{array}$ & Number & $\begin{array}{c}\text { miR-10b } \\
\text { expression }\end{array}$ & p-value \\
\hline \multicolumn{4}{|l|}{ Age (years) } \\
\hline$<60$ & 120 & $3.04 \pm 1.02$ & \multirow{2}{*}{0.948} \\
\hline$\geq 60$ & 74 & $3.03 \pm 1.07$ & \\
\hline \multicolumn{4}{|l|}{ Gender } \\
\hline Male & 113 & $2.93 \pm 1.10$ & \multirow{2}{*}{0.084} \\
\hline Female & 81 & $3.19 \pm 0.92$ & \\
\hline \multicolumn{4}{|l|}{ Smoking History } \\
\hline Yes & 117 & $3.17 \pm 0.99$ & \multirow{2}{*}{0.030} \\
\hline No & 77 & $2.84 \pm 1.08$ & \\
\hline \multicolumn{4}{|l|}{ Differentiation } \\
\hline Low-moderate & 80 & $3.30 \pm 0.98$ & \multirow[t]{2}{*}{0.003} \\
\hline High & 114 & $2.85 \pm 1.04$ & \\
\hline \multicolumn{4}{|l|}{ Distant metastasis } \\
\hline Yes & 102 & $2.51 \pm 0.91$ & \multirow{2}{*}{$<0.001$} \\
\hline No & 92 & $3.62 \pm 0.85$ & \\
\hline \multicolumn{4}{|l|}{ Lymph node metastasis } \\
\hline No & 86 & $2.59 \pm 0.94$ & \multirow{2}{*}{$<0.001$} \\
\hline Yes & 108 & $3.39 \pm 0.97$ & \\
\hline \multicolumn{4}{|l|}{ Pathological types } \\
\hline Squamous cell carcinoma & 61 & $2.66 \pm 1.02$ & \multirow{3}{*}{$<0.001$} \\
\hline Adenocarcinoma & 119 & $3.29 \pm 0.98$ & \\
\hline Other & 14 & $2.51 \pm 0.94$ & \\
\hline
\end{tabular}

Notes: NSCLC, non-small cell lung cancer; miR-10b, microRNA-10b.

Table 2. Influencing factors for the clinical response to chemotherapy for advanced NSCLC patients.

\begin{tabular}{|c|c|c|c|c|c|c|c|}
\hline \multirow{2}{*}{ Factor } & \multirow{2}{*}{ B } & \multirow{2}{*}{ SE } & \multirow{2}{*}{ Wald } & \multirow{2}{*}{ p-value } & \multirow{2}{*}{ OR } & \multicolumn{2}{|c|}{$95 \% \mathrm{CI}$} \\
\hline & & & & & & Lower & Upper \\
\hline Age & 0.297 & 0.503 & 0.350 & 0.554 & 1.346 & 0.502 & 3.608 \\
\hline Gender & 0.380 & 0.512 & 0.549 & 0.459 & 1.462 & 0.535 & 3.991 \\
\hline Differentiation & -0.740 & 0.558 & 1.760 & 0.185 & 0.477 & 0.160 & 1.424 \\
\hline Distant metastasis & 1.922 & 0.537 & 12.813 & $<0.001$ & 6.833 & 2.386 & 19.568 \\
\hline Lymph node metastasis & 1.871 & 0.499 & 14.088 & $<0.001$ & 6.495 & 2.445 & 17.255 \\
\hline Smoking history & 1.515 & 0.497 & 9.312 & 0.002 & 4.550 & 1.719 & 12.042 \\
\hline Pathological types & -0.304 & 0.413 & 0.540 & 0.462 & 0.738 & 0.328 & 1.659 \\
\hline MiR-10b expression & 2.795 & 0.579 & 23.282 & $<0.001$ & 16.361 & 5.257 & 50.919 \\
\hline
\end{tabular}

Notes: B, regression coefficient; SE, standard error; OR, odds ratio; CI, confidence interval; NSCLC, non-small cell lung cancer; miR-10b, microRNA-10b. 
A

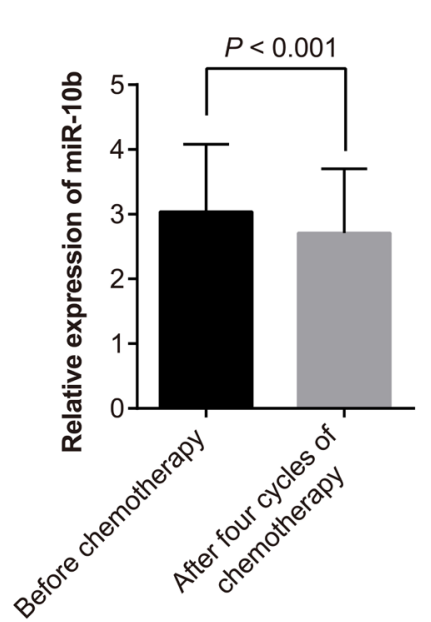

B

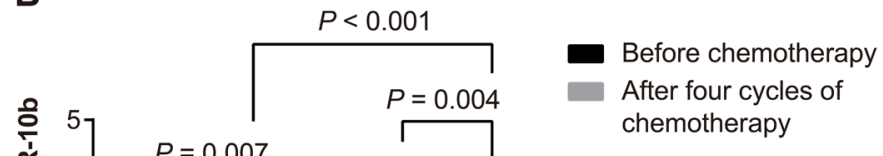

Figure 2. Expression of miR-10b in PBMCs of advanced NSCLC before chemotherapy and after four cycles of chemotherapy. A) expression of miR$10 \mathrm{~b}$ in PBMCs before chemotherapy and after four cycles of chemotherapy; $B$ ) expression of miR-10b in PBMCs in the effective group (CR+PR) and the ineffective group (PD+SD) before chemotherapy and after four cycles of chemotherapy, paired t-test was used to analyze the difference before chemotherapy and after four cycles of chemotherapy, unpaired $t$-test was used to analyze the difference between the effective group (CR+PR) and the ineffective group (PD+SD) after chemotherapy; NSCLC; non-small-cell lung cancer; PBMCs: peripheral blood mononuclear cells; CR, complete response; $\mathrm{PR}$, partial response; $\mathrm{PD}$, progressed disease; $\mathrm{SD}$, stable disease.

A

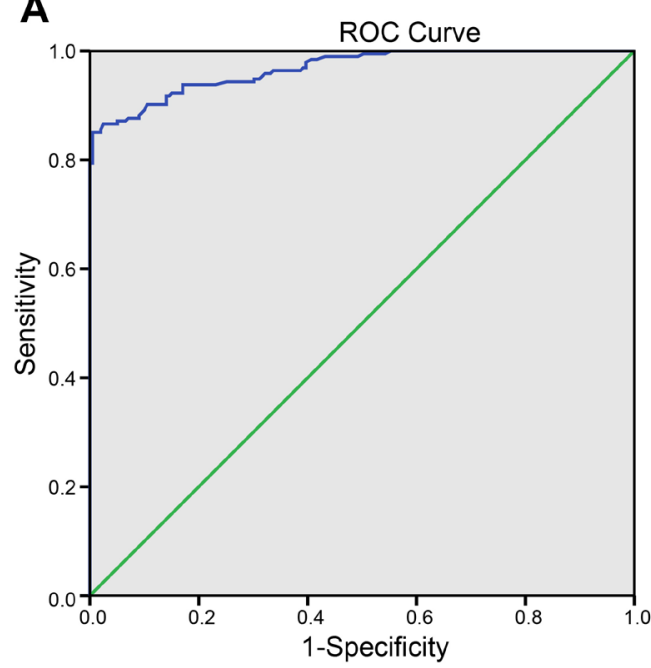

B

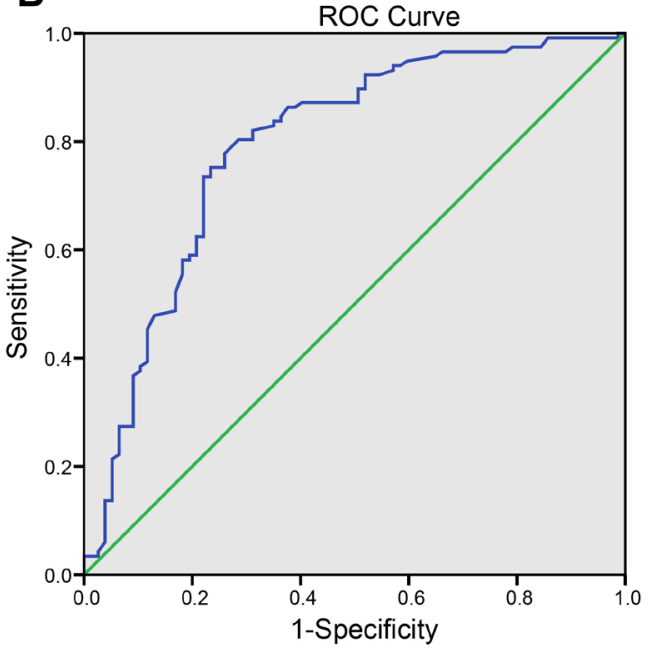

Figure 3. Predictive value of miR-10b expression in PBMCs for advanced NSCLC patients with chemotherapy. A) ROC curve of the predictive value of miR-10b expression for advanced NSCLC in the NSCLC and control groups; B) ROC curve of the predictive value of miR-10b expression in the clinical response to chemotherapy for advanced NSCLC in the effective and ineffective groups; ROC, receiver operating characteristic; miR-10b, microRNA10b; NSCLC, non-small-cell lung cancer; PBMCs: peripheral blood mononuclear cells.

Kaplan-Meier univariate analysis of influencing factors for the prognosis of patients with advanced NSCLC. Univariate analysis was performed with Kaplan-Meier survival curves, and Log-Rank test was applied to detect the difference of Kaplan-Meier survival curve between groups (Table 3). When the threshold value (2.87) of ROC curve served as the cutoff value of miR-10b expression, the NSCLC patients were classified into high expression group and low expression group. Kaplan-Meier survival analysis curve of miR-10b expression, smoking history, LNM and distant metastasis are shown in Figure 4. The results show that median PFS and 5-year survival rate were 12.0 months and $0 \%$ in patients with high miR-10b expression, respectively, which was prominently reduced compared to patients with low miR-10b expression (median PFS: 18.0 months; the 5-year survival rate: $19.1 \%$ ) (all $\mathrm{p}<0.05$ ). Median PFS 
Table 3. Kaplan-Meier univariate analysis of influencing factors for the prognosis of patients with advanced NSCLC.

\begin{tabular}{|c|c|c|c|c|c|c|}
\hline \multirow{2}{*}{ Clinicopathological data } & \multirow{2}{*}{ Number } & \multicolumn{2}{|c|}{ PFS } & \multicolumn{2}{|c|}{$95 \% \mathrm{CI}$} & \multirow{2}{*}{ p-value } \\
\hline & & Estimated value & SD & Lower & Upper & \\
\hline \multicolumn{7}{|l|}{ Age (years) } \\
\hline$<60$ & 120 & 14 & 0.855 & 12.324 & 15.676 & \multirow{2}{*}{0.956} \\
\hline$\geq 60$ & 74 & 13 & 0.644 & 11.737 & 14.263 & \\
\hline \multicolumn{7}{|l|}{ Gender } \\
\hline Male & 113 & 14 & 0.874 & 12.287 & 15.713 & \multirow{2}{*}{0.050} \\
\hline Female & 81 & 13 & 1.038 & 10.966 & 15.034 & \\
\hline \multicolumn{7}{|l|}{ Smoking history } \\
\hline Yes & 117 & 12 & 0.773 & 10.486 & 13.514 & \multirow{2}{*}{$<0.001$} \\
\hline No & 77 & 16 & 1.194 & 13.660 & 18.340 & \\
\hline \multicolumn{7}{|l|}{ Differentiation } \\
\hline Low-moderate & 114 & 13 & 1.051 & 10.940 & 15.060 & \multirow{2}{*}{0.096} \\
\hline High & 80 & 14 & 0.720 & 12.589 & 15.411 & \\
\hline \multicolumn{7}{|l|}{ Distant metastasis } \\
\hline No & 102 & 17 & 1.407 & 14.242 & 19.758 & \multirow{2}{*}{$<0.001$} \\
\hline Yes & 92 & 11 & 0.684 & 9.660 & 12.340 & \\
\hline \multicolumn{7}{|l|}{ Lymph node metastasis } \\
\hline No & 86 & 17 & 1.604 & 13.857 & 20.143 & \multirow{2}{*}{$<0.001$} \\
\hline Yes & 108 & 12 & 0.647 & 10.732 & 13.268 & \\
\hline \multicolumn{7}{|l|}{ Pathological types } \\
\hline Squamous cell carcinoma & 61 & 14 & 1.259 & 11.533 & 16.467 & \multirow{3}{*}{0.228} \\
\hline Adenocarcinoma & 119 & 13 & 0.818 & 11.397 & 14.603 & \\
\hline Others & 14 & 15 & 1.195 & 12.657 & 17.343 & \\
\hline \multicolumn{7}{|l|}{ MiR-10b } \\
\hline High & 89 & 12 & 0.590 & 10.843 & 13.157 & \multirow{2}{*}{$<0.001$} \\
\hline Low & 105 & 18 & 2.495 & 13.111 & 22.889 & \\
\hline
\end{tabular}

Notes: PFS, progression free survival; CI, confidence interval; SD, standard deviation; NSCLC, non-small cell lung cancer; miR-10b, microRNA-10b.

Table 4. Influencing factors for the prognosis of advanced NSCLC patients.

\begin{tabular}{|c|c|c|c|c|c|c|c|}
\hline \multirow{2}{*}{ Factors } & \multirow{2}{*}{ B } & \multirow{2}{*}{ SE } & \multirow{2}{*}{ Wald } & \multirow{2}{*}{ p-value } & \multirow{2}{*}{ RR } & \multicolumn{2}{|c|}{$95 \% \mathrm{CI}$} \\
\hline & & & & & & Lower & Upper \\
\hline Distant metastasis & 0.532 & 0.205 & 6.772 & 0.009 & 1.703 & 1.140 & 2.543 \\
\hline Lymph node metastasis & 0.416 & 0.175 & 5.672 & 0.017 & 1.515 & 1.076 & 2.133 \\
\hline Smoking history & 0.425 & 0.164 & 6.713 & 0.010 & 1.529 & 1.109 & 2.108 \\
\hline MiR-10b & 0.571 & 0.210 & 7.358 & 0.007 & 1.769 & 1.172 & 2.672 \\
\hline
\end{tabular}

Notes: B, regression coefficient; SE, standard error; RR, relative risk; CI, confidence interval; NSCLC, non-small cell lung cancer; miR-10b, microRNA-10b.

and 5-year survival rate were 15.0 months and $14.3 \%$ in patients without smoking history, respectively, which were dramatically elevated compared to patients with smoking history (median PFS: 13.0 month; the 5-year survival rate: $5.1 \%)($ all $\mathrm{p}<0.05)$. Median PFS and 5-year survival rate were 17.0 month and $17.4 \%$ in patient without LNM, respectively, which were evidently enhanced compared to patients with LNM (median PFS: 12.0 month; the 5-year survival rate: 1.9\%) (all $\mathrm{p}<0.05)$. Median PFS and 5-year survival rate were 17.0 months and $16.7 \%$ in patients without distant metastasis, respectively, and both were obviously ascended compared to patients with distant metastasis (median PFS: 11.0 month; the 5-year survival rate: $0 \%)($ all $\mathrm{p}<0.05)$. Therefore, miR-10b expression, smoking history, LNM and distant metastases were predictive factors for PFS for advanced NSCLC patients after chemotherapy.

Cox proportional hazard regression analysis for the risk factors of the prognosis for patients with advanced NSCLC. Statistically significant factors (miR-10b expression, smoking history, LNM and distant metastasis) were included in the Cox proportional hazard model as seen in Table 4. The results showed that the over-expression of miR-10b, smoking history, LNM and distant metastasis were prognosis risk factors for patients with advanced NSCLC (all $\mathrm{p}<0.05)$. 
A

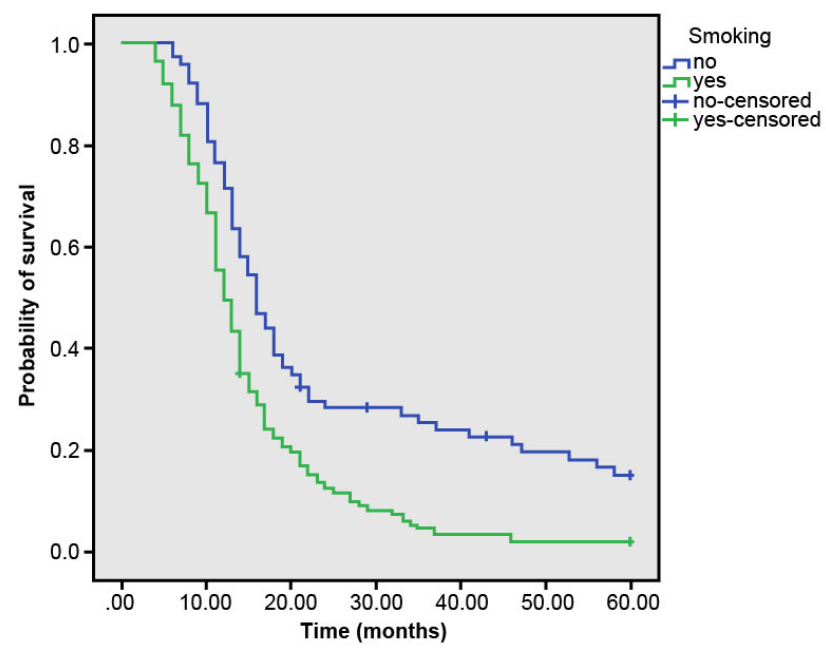

C

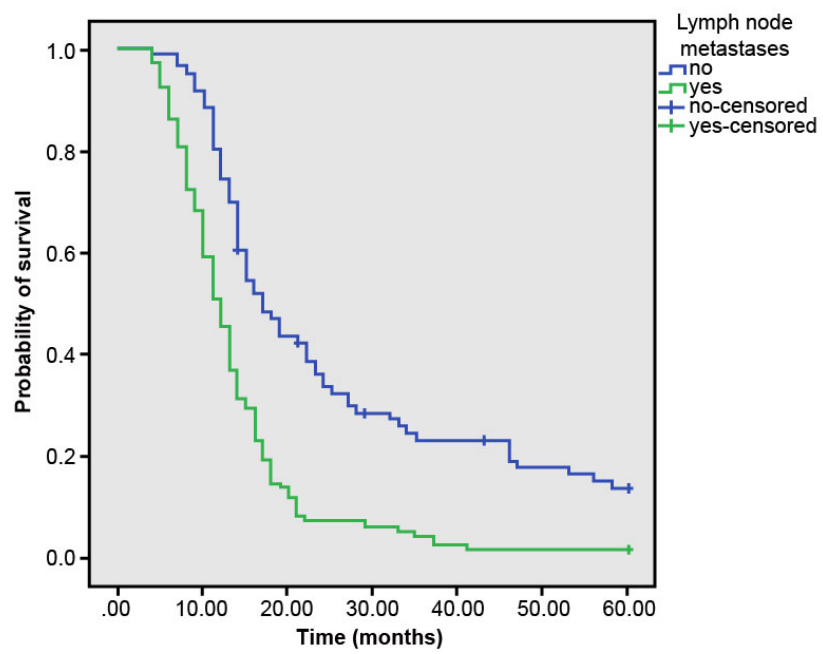

B

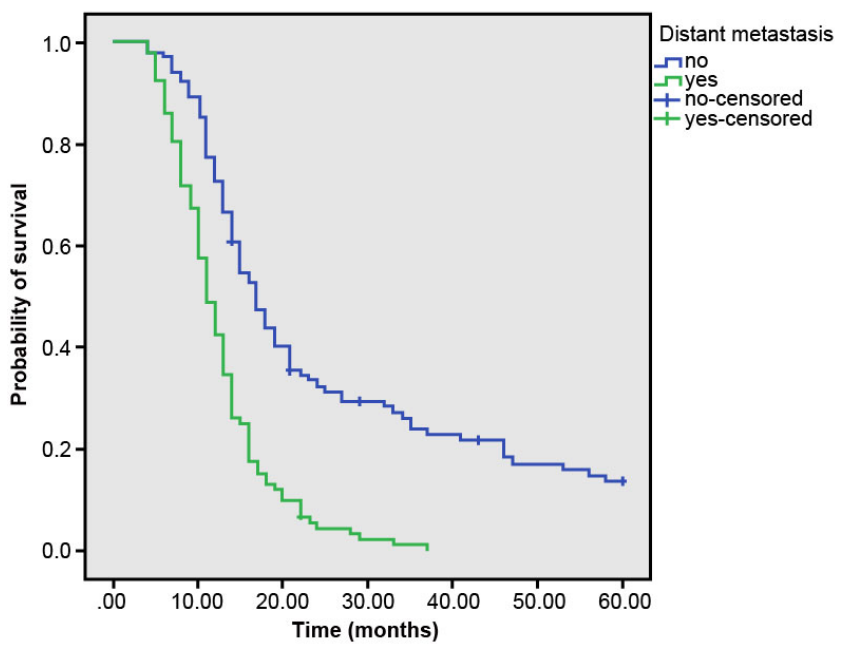

D

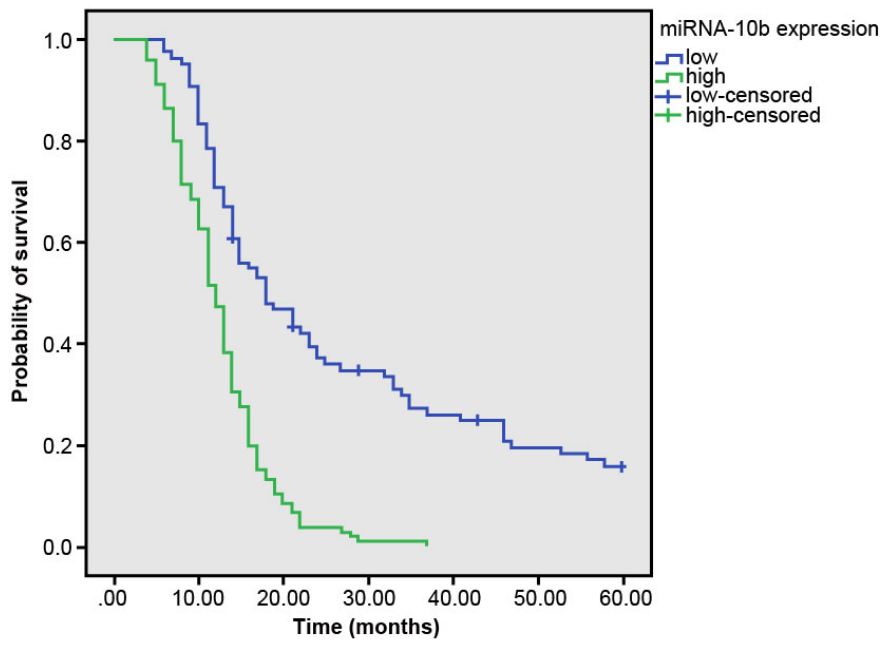

Figure 4. Kaplan-Meier survival curves of influencing factors for the PFS of advanced NSCLC patients. A) Kaplan-Meier survival curves of the PFS of advanced NSCLC patients with smoking history; B) Kaplan-Meier survival curves of the PFS of advanced NSCLC patients with distant metastasis; C) Kaplan-Meier survival curves of the PFS of advanced NSCLC patients with LNM; D) Kaplan-Meier survival curves of the PFS of advanced NSCLC patients in the low expression and high expression miR-10b groups; LNM, lymph node metastasis; miR-10b, microRNA-10b; PFS, progression free survival; NSCLC, non-small cell lung cancer.

\section{Discussion}

NSCLC is one of the most common lung cancers, and finding effective methods of treatment is very important for NSCLC patients [21]. Further research is necessary to improve the diagnosis, early prevention, and treatments to cure this cancer and more and more studies have shown that miRs may play a decisive role in NSCLC pathogenesis $[14,22,23]$. In our study, we investigated predictive value of miR-10b expression in PBMCs in evaluating clinical response to chemotherapy and prognosis for patients with advanced NSCLC. The results of present study indicate that over-expression of miR-10b in PBMCs could predict worse tumor response to chemotherapy and prognosis for advanced NSCLC patients.

Initially, our study demonstrated that miR-10b expression in PBMCs in the NSCLC group was significantly higher than that in the control group and over-expression of miR-10b was closely related to poorer outcomes. The abnormal expression of several miRs have been proven to serve as important biomarkers for the diagnosis, prognosis and survival [24]. Consistent with our results, Zhang et al. reported that miR-10b, which is involved in cancer invasion and metastasis, is up-regulated in the NSCLC cells and 
tissues, and even the overexpression predicts poor prognosis [25]. Besides, it has been proven that up-regulated miR-10b in breast cancer cells promotes the invasion and metastasis of tumor cells with an increase in the metastatic potential, thereby leads to a poor prognosis $[26,27]$. Furthermore, miR-10b is over-expressed in pancreatic cancer cells and is related to prognosis of pancreatic cancer patients [13]. As an oncogene, the overexpression of miR-10b in the metastatic lymph node tissues results in metastasis development in the NSCLC, indicating a key role of miR-10b in the lung cancer cell metastasis [28]. As reflected in our study, over-expressed miR-10b in the PBMCs of patients with advanced NSCLC is strongly correlated with LNM, distant metastasis, low-moderate differentiation, squamous cell carcinoma and stage IV cancer. In line with the finding, miR-10b was differently expressed in squamous cell carcinoma (SCC) and $\mathrm{AC}$, indicating a correlation of miR-10b with the clinical stage of NSCLC [29]. Additionally, the report suggests the up-regulated expression of miR-10b is closely related to a larger tumor size as well as advanced pathological stage of NSCLC, and the inhibition of miR-10b might provide a novel way for the clinical diagnosis and targeted therapy [30]. A previous study has revealed that overexpression of miR-10b enhances cell proliferation, migration, and invasion capacities in NSCLC, and Kruppel-like factor 4 (KLF4) may be indirectly targeted by miR-10b during the increased proliferation of NSCLC cells [14]. Another relevant study has identified that miR-10b targets KLF4 to affect the biological function of cells in human esophageal cancer, thereby affects the progression, metastasis and prognosis of tumor [31]. By downregulating the tumor suppressor gene KLF4, miR-10b can promote cancer cell proliferation and invasive metastasis, possibly by preventing the KLF4-induced G1/S cell cycle arrest through the regulation of the extracellular matrix proteins, cyclin D1 or p21 expression [32]. Meanwhile, the miR-10b expression might negatively regulate the E-cadherin expression (a potent suppressor of lung cancer metastasis), which directly affects the tumor grade and stage, to function as an independent factor for the prognosis of NSCLC patients [25]. Yang et al. have also reported that miR-10b expression in the PBMCs isolated from NSCLC patients showed high diagnostic sensitivity and specificity; high miR-10b expression, patients' age, LNM and distant metastases were independent risk factors for worse prognosis of NSCLC patients [7], which is consistent with the results of the logistic regression analysis, univariate analysis and ROC curves in the presented paper. All the data above contribute to a conclusion that miR-10b is an important diagnostic and prognostic factor for patients with advanced NSCLC.

Additionally, compared with the expression of miR-10b before chemotherapy, miR-10b expression was significantly decreased after four cycles of chemotherapy; and the effective group exhibited strikingly lower miR-10b expression than the ineffective group. The plasma miRNAs (hsa-miR-98-5p, hsa-miR-302e, hsa-miR-495-3p, and hsa-miR-613) in NSCLC patients are important predictors of the radiosensitivity, and became novel biomarkers for radiotherapy response [33]. Besides, the circulating cell-free miRs in blood plasma and serum are noted to be associated with the tumor progression and prognosis of lung cancer, and serve as tumor biomarkers and prognosticators in patients with NSCLC or other types of lung cancers [34]. In the presented study, we showed that miR-10b is not only associated with tumor response to chemotherapy after a four-course treatment, but is also related to the PFS and survival rate after follow-ups. Although several miRs are involved in the chemoresistance of NSCLC cells to cisplatin, such as miR-182 and miR-192 $[35,36]$, miR-10b was demonstrated to be a potential indicator of chemosensitivity in colorectal cancer [11]. It still remains to determine the value of miR-10b in predicting the efficacy of chemotherapy for other cancers. We suggest that miR-10b expression in PBMCs has a high predictive value for the efficacy of chemotherapy for patients with advanced NSCLC, both in the short-term tumor response and long-term survival.

In summary, we provide strong evidence that high miR-10b expression in PBMCs of NSCLC patients correlates with poor prognosis and it can effectively predict tumor response to chemotherapy (short-term efficacy) and prognosis (long-term efficacy) for advanced NSCLC patients. Nevertheless, a larger population is needed to further identify whether other aberrantly expressed miRs interfere with these results and the specific mechanisms of miR-10b in the chemosensitivity may be further explored in future studies, including critical signaling pathways or essential factors related to the treatment of lung cancer, such as epidermal growth factor receptor (EGFR), anaplastic lymphoma kinase (ALK), etc.

\section{References}

[1] ZHANG WC, SHYH-CHANG N, YANG H, RAI A, UMASHANKAR $S$ et al. Glycine decarboxylase activity drives non-small cell lung cancer tumor-initiating cells and tumorigenesis. Cell 2012; 148: 259-272. https://doi. org/10.1016/j.cell.2011.11.050

[2] ROTH JA, CARLSON JJ. Prognostic role of ERCC1 in advanced non-small-cell lung cancer: a systematic review and meta-analysis. Clin Lung Cancer 2011; 12: 393-401. https:// doi.org/10.1016/j.cllc.2011.04.005

[3] BOKOBZA SM, JIANG Y, WEBER AM, DEVERY AM, RYAN AJ. Combining AKT inhibition with chloroquine and gefitinib prevents compensatory autophagy and induces cell death in EGFR mutated NSCLC cells. Oncotarget 2014; 5: 4765-4778. https://doi.org/10.18632/oncotarget.2017

[4] GOULART BH, REYES CM, FEDORENKO CR, MUMMY DG, SATRAM-HOANG $S$ et al. Referral and treatment patterns among patients with stages III and IV non-smallcell lung cancer. J Oncol Pract 2013; 9: 42-50. https://doi. org/10.1200/JOP.2012.000640 
[5] OGAWA F, WANG G, MATSUI Y, HARA H, IYODA A et al. Risk factors for postoperative complications in the elderly with lung cancer. Asian Cardiovasc Thorac Ann 2013; 21: 313-318. https://doi.org/10.1177/0218492312457359

[6] WANG T, NELSON RA, BOGARDUS A, GRANNIS FW, JR. Five-year lung cancer survival: which advanced stage nonsmall cell lung cancer patients attain long-term survival? Cancer 2010; 116: 1518-1525. https://doi.org/10.1002/cncr.24871

[7] YANG YL, XU LP, ZHUO FL, WANG TY. Prognostic value of microRNA-10b overexpression in peripheral blood mononuclear cells of nonsmall-cell lung cancer patients. Tumour Biol 2015; 36: 7069-7075. https://doi.org/10.1007/s13277015-3366-6

[8] JIANG J, LV X, FAN L, HUANG G, ZHAN Y et al. MicroRNA-27b suppresses growth and invasion of NSCLC cells by targeting Sp1. Tumour Biol 2014; 35: 10019-10023. https:// doi.org/10.1007/s13277-014-2294-1

[9] CUI EH, LI HJ, HUA F, WANG B, MAO W et al. Serum microRNA $125 \mathrm{~b}$ as a diagnostic or prognostic biomarker for advanced NSCLC patients receiving cisplatin-based chemotherapy. Acta Pharmacol Sin 2013; 34: 309-313. https://doi. org/10.1038/aps.2012.125

[10] FOSS KM, SIMA C, UGOLINI D, NERI M, ALLEN KE et al. miR-1254 and miR-574-5p: serum-based microRNA biomarkers for early-stage non-small cell lung cancer. J Thorac Oncol 2011; 6: 482-488. https://doi.org/10.1097/ JTO.0b013e318208c785

[11] NISHIDA N, YAMASHITA S, MIMORI K, SUDO T, TANAKA F et al. MicroRNA-10b is a prognostic indicator in colorectal cancer and confers resistance to the chemotherapeutic agent 5-fluorouracil in colorectal cancer cells. Ann Surg Oncol 2012; 19: 3065-3071. https://doi.org/10.1245/ s10434-012-2246-1

[12] MA L. Role of miR-10b in breast cancer metastasis. Breast Cancer Res 2010; 12: 210. https://doi.org/10.1186/bcr2720

[13] NAKATA K, OHUCHIDA K, MIZUMOTO K, KAYASHIMA T, IKENAGA $\mathrm{N}$ et al. MicroRNA-10b is overexpressed in pancreatic cancer, promotes its invasiveness, and correlates with a poor prognosis. Surgery 2011; 150: 916-922. https://doi.org/10.1016/j.surg.2011.06.017

[14] LIU Y, LI M, ZHANG G, PANG Z. MicroRNA-10b overexpression promotes non-small cell lung cancer cell proliferation and invasion. Eur J Med Res 2013; 18: 41. https://doi. org/10.1186/2047-783X-18-41

[15] MA J, LIN Y, ZHAN M, MANN DL, STASS SA et al. Differential miRNA expressions in peripheral blood mononuclear cells for diagnosis of lung cancer. Lab Invest 2015; 95: $1197-$ 1206. https://doi.org/10.1038/labinvest.2015.88

[16] WANG WS, LIU LX, LI GP, CHEN Y, LI CY et al. Combined serum CA19-9 and miR-27a-3p in peripheral blood mononuclear cells to diagnose pancreatic cancer. Cancer Prev Res (Phila) 2013; 6: 331-338. https://doi.org/10.1158/1940-6207. CAPR-12-0307

[17] ZHANG B, LIU T, WU T, WANG Z, RAO Z et al. microRNA-137 functions as a tumor suppressor in human nonsmall cell lung cancer by targeting SLC22A18. Int J Biol Macromol 2015; 74: 111-118. https://doi.org/10.1016/j. ijbiomac.2014.12.002
[18] WANG Y, HA M, LIU J, LI P, ZHANG W et al. Role of BCL2associated athanogene in resistance to platinum-based chemotherapy in non-small-cell lung cancer. Oncol Lett 2016; 11: 984-990. https://doi.org/10.3892/ol.2015.4003

[19] PANG L, WANG J, JIANG Y, CHEN L. Decreased levels of serum cytokeratin 19 fragment CYFRA 21-1 predict objective response to chemotherapy in patients with non-small cell lung cancer. Exp Ther Med 2013; 6: 355-360. https://doi. org/10.3892/etm.2013.1171

[20] EISENHAUER EA, THERASSE P, BOGAERTS J, SCHWARTZ LH, SARGENT D et al. New response evaluation criteria in solid tumours: revised RECIST guideline (version 1.1). Eur J Cancer 2009; 45: 228-247. https://doi. org/10.1016/j.ejca.2008.10.026

[21] WU H, ZHONG Q, ZHONG R, HUANG H, XIA Z et al. Preparation and antitumor evaluation of self-assembling oleanolic acid-loaded Pluronic P105/d-alpha-tocopheryl polyethylene glycol succinate mixed micelles for non-smallcell lung cancer treatment. Int J Nanomedicine 2016; 11: 6337-6352. https://doi.org/10.2147/IJN.S119839

[22] ARORA S, RANADE AR, TRAN NL, NASSER S, SRIDHAR $S$ et al. MicroRNA-328 is associated with (non-small) cell lung cancer (NSCLC) brain metastasis and mediates NSCLC migration. Int J Cancer 2011; 129: 2621-2631. https://doi. org/10.1002/ijc.25939

[23] CHEN L, JIN H. MicroRNAs as novel biomarkers in the diagnosis of non-small cell lung cancer: a meta-analysis based on 20 studies. Tumour Biol 2014; 35: 9119-9129. https://doi. org/10.1007/s13277-014-2188-2

[24] GALASSO M, SANA ME, VOLINIA S. Non-coding RNAs: a key to future personalized molecular therapy? Genome Med 2010; 2: 12. https://doi.org/10.1186/gm133

[25] ZHANG J, XU L, YANG Z, LU H, HU D et al. MicroRNA$10 \mathrm{~b}$ indicates a poor prognosis of non-small cell lung cancer and targets E-cadherin. Clin Transl Oncol 2015; 17: 209-214. https://doi.org/10.1007/s12094-014-1213-7

[26] WU ZS, WANG CQ, XIANG R, LIU X, YE S et al. Loss of miR-133a expression associated with poor survival of breast cancer and restoration of miR-133a expression inhibited breast cancer cell growth and invasion. BMC Cancer 2012; 12: 51. https://doi.org/10.1186/1471-2407-12-51

[27] AHMAD A, SETHI S, CHEN W, ALI-FEHMI R, MITTAL S et al. Up-regulation of microRNA-10b is associated with the development of breast cancer brain metastasis. Am J Transl Res 2014; 6: 384-390.

[28] LI Y, LI Y, LIU J, FAN Y, LI X et al. Expression levels of microRNA-145 and microRNA-10b are associated with metastasis in non-small cell lung cancer. Cancer Biol Ther 2016; 17: 272-279. https://doi.org/10.1080/15384047.2016.1139242

[29] SKRZYPSKI M, CZAPIEWSKI P, GORYCA K, JASSEM E, WYRWICZ L et al. Prognostic value of microRNA expression in operable non-small cell lung cancer patients. Br J Cancer 2014; 110: 991-1000. https://doi.org/10.1038/ bjc. 2013.786

[30] HUANG J, SUN C, WANG S, HE Q, LI D. microRNA miR$10 \mathrm{~b}$ inhibition reduces cell proliferation and promotes apoptosis in non-small cell lung cancer (NSCLC) cells. Mol Biosyst 2015; 11: 2051-2059. https://doi.org/10.1039/ $\mathrm{c} 4 \mathrm{mb} 00752 \mathrm{~b}$ 
[31] TIAN Y, LUO A, CAI Y, SU Q, DING F et al. MicroRNA-10b promotes migration and invasion through KLF4 in human esophageal cancer cell lines. J Biol Chem 2010; 285: 79867994. https://doi.org/10.1074/jbc.M109.062877

[32] SU QL, LI SQ, WANG DN, LIU F, YUAN B. Effects of MicroRNA-10b on lung cancer cell proliferation and invasive metastasis and the underlying mechanism. Asian Pac J Trop Med 2014; 7: 364-367. https://doi.org/10.1016/S19957645(14)60056-0

[33] CHEN X, XU Y, LIAO X, LIAO R, ZHANG L et al. Plasma miRNAs in predicting radiosensitivity in non-small cell lung cancer. Tumour Biol 2016; 37: 11927-11936. https://doi. org/10.1007/s13277-016-5052-8
[34] SCHWARZENBACH H, NISHIDA N, CALIN GA, PANTEL $\mathrm{K}$. Clinical relevance of circulating cell-free microRNAs in cancer. Nature reviews Clinical oncology 2014; 11: 145-156.

[35] NING FL, WANG F, LI ML, YU ZS, HAO YZ et al. MicroRNA-182 modulates chemosensitivity of human non-small cell lung cancer to cisplatin by targeting PDCD4. Diagn Pathol 2014; 9: 143. https://doi.org/10.1186/1746-1596-9-143

[36] CAO J, HE Y, LIU HQ, WANG SB, ZHAO BC et al. MicroRNA 192 regulates chemo-resistance of lung adenocarcinoma for gemcitabine and cisplatin combined therapy by targeting Bcl-2. Int J Clin Exp Med 2015; 8: 12397-12403. 